\title{
Cultura digital emergente y prácticas educativas expandidas: \\ reflexiones desde Platohedro*
}

\author{
Emerging \\ Digital Culture \\ and Expanded \\ Educational \\ Practices: \\ Reflections from \\ Platohedro
}

Primer semestre de 2020 - pp. 117-127 Segunda época

N. 0

\author{
Cultura digital \\ emergente \\ e práticas \\ educacionais \\ ampliadas: \\ reflexões do \\ Platohedro
}

Para citar este artículo

Uribe, A. (2020). Cultura digital emergente y prácticas educativas expandidas: reflexiones desde Platohedro. Folios, 51, 117-127. doi: 10.17227/folios.51-9577

Artículo recibido $01 \cdot 02 \cdot 2019$

Artículo aprobado $25 \cdot 07 \cdot 2019$

Ciencia, Tecnología e Innovación de Colombia.

** Doctor en Educación de la Universidad de Antioquia., Medellín, Colombia. Profesor de la Escuela de Educación y Pedagogía de la Universidad Pontificia Bolivariana y miembro del Grupo de Investigación EAV, Medellín, Colombia.

Correo electrónico: alejandro.uribe@upb.edu.co. 


\section{Resumen}

Este artículo de reflexión explora las relaciones entre las categorías de hibridez, agencia, socialización, farmacón y decolonización en el marco de una época posdigital y una práctica educativa expandida. Las categorías emergieron después de un trabajo de campo realizado durante el 2016 en Platohedro, una iniciativa de la ciudad de Medellín, Colombia. La investigación tuvo un enfoque cualitativo, se apoyó en una versión constructivista de la teoríaada y uno de los objetivos era comprender la emergencia de algunas prácticas ciudadanas juveniles que usan de manera intensiva internet. Se cierra el artículo presentando algunas implicaciones educativas.

\section{Palabras clave}

pedagogía; educación; educación expandida; cultura digital

\section{Abstract}

This reflection paper explores the relationship between the categories of hybridity, agency, socialization, pharmacon and decoloniality, within the framework of a post-digital era and an expanded educational practice. These categories emerged after a fieldwork developed in 2016 in Platohedro - an initiative from Medellín, Colombia. The research had a qualitative approach, based on a constructivist version of the grounded theory. One of its objectives was to comprehend the emergence of some juvenile practices of citizenship, which use Internet intensively. Some educational implications are presented at the end of the article.

\section{Keywords}

pedagogy; education; expanded education; digital culture

\section{Resumo}

Este artigo de reflexão explora as relações entre as categorias de hibridismo, agência, socialização, farmacon e descolonização no quadro de uma era pós-digital e uma prática educacional expandida. As categorias surgiram após um trabalho de campo realizado em 2016 no Platohedro, uma iniciativa da cidade de Medellín, Colômbia. A pesquisa teve uma abordagem qualitativa, baseou-se em uma versão construtivista da teoria fundamentada e um dos objetivos foi compreender o surgimento de algumas práticas de jovens cidadãos que usa; intensament; a Internet. 0 artigo apresenta algumas implicações educacionais no final.

\section{Palavras-chave}

pedagogia; educação; educação ampliada; cultura digital 


\section{Introducción}

En general, cuando se habla de cultura digital, los principales elementos que aparecen asociados a este término son la participación, la remediación y el bricolaje (Deuze, 2006). En el primer caso, lo participativo denota la cultura que adopta los valores de diversidad y democracia para cada una de nuestras interacciones; considera que, como individuos y sociedad, tenemos la capacidad de tomar decisiones propias, y defiende la posibilidad de expresarnos de manera libre a través de un amplio abanico de formas y prácticas (Jenkins, Itō, \& boyd, 2016). En un texto anterior, Jenkins y otro grupo de colegas, presentan cinco rasgos propios de esta cultura de la participación: 1) hay pocas barreras para la expresión artística y el compromiso ciudadano; 2) apoya con firmeza la creación y el intercambio con los demás; 3) la experticia informal se distribuye entre los aficionados y los más novatos; 4) los miembros consideran que sus contribuciones tienen relevancia y 5) establecen en cierta medida conexiones sociales con los demás (Jenkins, Purushotma, Weigel, Clinton y Robison, 2009).

En el segundo caso, la remediación se refiere a la modificación, manipulación y reformulación de la información disponible. En términos epistémicos, es la posibilidad de reconfigurar y alterar la realidad. En contextos de mediación digital, donde los recursos son potencialmente adaptables al gusto individual, se espera que las personas construyan y divulguen sus propias reflexiones y versiones de los hechos antes de asumir pasivamente los discursos oficialistas, institucionalizados y mainstream. Los blogs, las páginas de noticias independientes, los generadores de contenido en diversas plataformas, entre otros, ejemplifican esta corriente. En la red, tales posturas divergentes se validan y legitiman a través de los datos cuantificados que posibilitan este mismo espacio, a saber, número de comentarios, fans, trinos, me gusta, entre otros.

Finalmente, el bricolaje caracteriza a los que obran sin seguir un recetario, apelan a medios, recursos y procedimientos alejados de lo estándar, se acomodan a lo que hay y no trabajan con materias primarias sino con insumos ya elaborados, fragmentos, sobras y trozos de otras obras (Lévi-Strauss, 1997). En el marco de la cultura digital, esa idea se enriquece con prácticas e ideas antaño disruptivas, pero ya relativamente cotidianas como la redifusión de contenidos, la hibridez, la artesanía, la mezcla y remezcla, y el copie y pegue que posibilitan, para algunos, amplificar el sentido creado mediante la referencia para construir algo nuevo (Lessig, 2012) o estimular nuevas conexiones ya que funciona como un pegamento o aglutinador cultural (cultural glue) (Navas, 2012).

No obstante, otros autores plantean que ya estamos inmersos en una época posdigital (Cramer, 2015), no en el sentido de superar lo digital ni tampoco en el de estar, desde una perspectiva hegeliana, lineal, progresista y teleológica, en una fase histórica posterior, sino que se trata de subrayar cambios culturales y mutaciones que se están presentando en la actualidad luego de la creciente influencia generada por las tecnologías digitales en múltiples ámbitos de la vida. En contravía del fervor inicial de los primeros años de la red, lo posdigital implica, pero no se reduce, rescatar una mirada escéptica, pausada e incluso desencantada frente lo digital.

Las prácticas educativas expandidas (Uribe, 2018), también llamadas laboratorios ciudadanos (Ricaurte, 2018), son un ejemplo de lo anterior. Estas iniciativas se caracterizan por escapar de las clásicas etiquetas de formal, no formal e informal; por configurarse como movimientos o colectivos minoritarios de origen ciudadano que apelan a formas organizativas modulares y reticulares; promover prácticas educativas emergentes y roles formativos horizontales; difuminar en sus configuraciones pedagógicas las fronteras disciplinares; rescatar elementos minimizados por la modernidad como los afectos, los cuidados y la hospitalidad; apelar al principio pedagógico del aprender haciendo; abrazar la narrativa del prototipado; mostrar sensibilidad hacia los saberes populares y ancestrales y poner en tensión los dualismos modernos tales como expertos/novatos o digital/análogo. Para ejemplos concretos, sobre 
todo a nivel local, nacional e iberoamericano véase Uribe Zapata (2017), Fonseca Díaz (2011) y Díaz y Freire (2012).

Además de naturalizar los elementos fundacionales de la denominada cultura digital, léase participación, remediación y bricolaje, estas prácticas revisitan y robustecen dicha cultura al incluir referentes como la hibridez, la agencia, la socialización, el farmacón y la decolonialidad. Configuran una época posdigital o cultura digital emergente. En nuestro caso, estas últimas categorías emergieron después de un trabajo de campo realizado durante el primer semestre del 2016 en Platohedro ${ }^{1}$, una práctica educativa expandida ubicada en la zona oriente de la ciudad de Medellín. Dicho trabajo se realizó en el marco de la realización de la tesis doctoral del autor y donde uno de los objetivos era comprender la emergencia de algunas prácticas ciudadanas juveniles que usan de manera intensiva internet.

Sobre la iniciativa, Platohedro suele presentarse a sí misma como un espacio autogestionado de corte creativo, comunitario y colaborativo que se dedica a la investigación, experimentación y la autoformación de la mano de tecnologías libres que sirven para la creación, publicación y distribución de sus contenidos. Sobre los participantes, tres (3) son hombres y tres (3) son mujeres, y la mitad (3) tiene menos de treinta (30) años y la otra mitad (3) son mayores de cuarenta y uno (41). Sobre la investigación, tuvo un enfoque cualitativo y se apoyó en una versión constructivista de la teoría fundamentada (Charmaz, 2008). Esta versión evita emplear categorías predeterminadas ya que sostiene que la relación con la realidad es múltiple, que el proceso de investigación emerge a partir de la interacción constante con los datos. Se debe tener en cuenta la posición del investigador, así como la de los participantes; y que la investigación no es una tabula rasa, sino que ésta dialoga con algunas preconcepciones teóricas. Fuera de los referentes derivados de la literatura académica, las dos fuentes de información

1 Véase http://platohedro.org/ fueron la observación participante y las entrevistas semiestructuradas.

El propósito de este artículo es explorar las relaciones entre las categorías emergentes (hibridez, agencia, socialización, farmacón y decolonialidad) y la práctica educativa expandida concreta que las inspiró, Platohedro. Se cierra el texto con algunas implicaciones de corte educativo derivadas de tales categorías.

\section{Hibridez}

Antes pensábamos qué es lo virtual y lo real. Ahora pensamos que no es tan así, están juntos. Entonces la huertita y su página web, están dialogando con otras vueltas, con otros proyectos. Se potencia mucho más la red. Ahora a Platohedro viene una artista filipina en 2 meses, ¿y cómo se iba a enterar de todo esto si no fuera por toda esa dinamización online? (Luciana, comunicación personal, 23 de junio de 2016).

Esa distinción en el ámbito tecnológico entre lo nuevo y viejo, como si fueran dos compartimentos separados que no dialogan entre sí, es bastante imprecisa en el presente. Es una oposición obsoleta. Basta con ver las hibridaciones que realizan, en primera instancia, los artistas y diseñadores al conjugar programas avanzados de escritorio con técnicas manuales tales como los bocetos o el dibujo; y los intelectuales, escritores y académicos que usan máquinas de escribir y toman notas en papel, pero difunden vía internet sus producciones impresas. En segunda instancia, las prácticas que subvierten por completo las intenciones originales de diseño de un producto, por ejemplo, usar partes de un móvil para crear sensores, convertir un computador portátil en un servidor o usar tecnologías pensadas en principio para la comunicación como herramientas políticas y para el ejercicio ciudadano.

A pesar de vivir en un mundo mediatizado en exceso, actualmente hay una vuelta a las máquinas de escribir, celulares sin pantallas táctiles, discos de acetato, técnicas de impresión artesanales, libretas para tomar notas, bicicletas para la movilidad e incluso un creciente gusto de parte de los jóvenes por 
los trabajos manuales (Ocejo, 2017). Tales iniciativas no se consideran hoy en día como algo de la vieja guardia, excéntrico o desactualizado. Al contrario, son decisiones conscientes, tomadas por personas y colectivos bastante familiarizados con lo digital, que basan sus decisiones en lo que necesitan o quieren en cada caso puntual.

Ambas parcelas, lo digital y analógico, los átomos y los bits, no están separadas ni tienen características exclusivas, sino que se entrecruzan de manera permanente y alimentan las prácticas humanas. Siguiendo la clásica definición de Canclini frente a la hibridación, se trata de explicitar que somos testigos cada vez más de procesos socioculturales en el que estructuras o prácticas discretas (que tampoco son estrictamente puras sino igualmente mestizas), que existían en forma separada, se combinan para generar nuevas estructuras, objetos y prácticas (García Canclini, 2001).

Platohedro, como espacio híbrido, se opone a los dualismos. Busca romper con el mito de las escisiones radicales de corte moderno tales como viejo/nuevo, análogo/digital, átomos/bits o racional/ afectivo. Varias de sus prácticas conjugan todo lo anterior y por ello generan dinámicas propias. Sin duda, sus prácticas devienen mestizas e híbridas (véase figura 1). Procesos en los que la tecnología se vuelve invisible ya que las discusiones no giran sobre $x$ o $y$ herramienta, sino sobre lo que se quiere hacer más allá del recurso empleado (por ejemplo, hacer animaciones o instalaciones sonoras); los saberes populares y ancestrales se conjugan con ejercicios de programación o electrónica y las manos, no solo la cabeza, se unen a los afectos y las confidencias entre personas con formación variada y de diversa edad ${ }^{2}$. Pero al ser mestizas esas prácticas no son puras. Es decir, se alejan de los formatos y los cronogramas herméticos, no impera el silencio, la duración de las sesiones es un misterio en ocasiones, se mezcla lo magistral con lo dialógico, varios ejercicios no resultan, no se esconden los experimentos fallidos y los

2 Por ejemplo, véase http://platohedro.org/plantas-yperformance-post-antropocentrica-un-cuaderno-decreacion-para-devenir-planta/ procesos, antes que los resultados, marcan el compás. De ahí, precisamente, su potencia educativa.

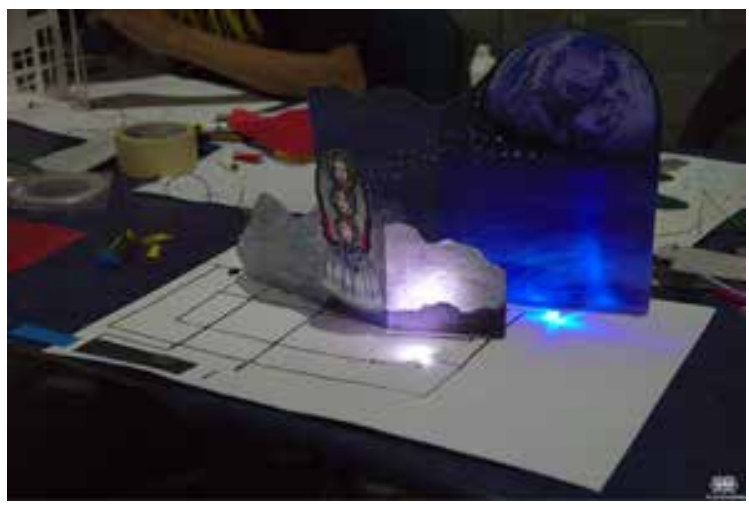

Figura 1. Taller de electrónica con papel. Fuente: Archivo Platohedro

\section{Agencia}

La ficción de la agencia, entendida de manera simple como la ilusión de control sobre el medio, se refuerza en el contexto de la cultura digital. Un ejemplo es el emergente movimiento DIY/DIWo/DITO (en castellano, significan hazlo tú mismo, hazlo con otros y hacerlo juntos $)^{3}$, que se remonta varias décadas atrás y pone en entredicho las dicotomías entre nuevo/ viejo y análogo/digital. La filosofía y las prácticas del tipo DIY/DIwo/dito ahora habitan en los espacios con impresoras 3D y otros dispositivos/herramientas para la creación, en los hackerspaces, makerspaces, en iniciativas con Arduino y software/hardware libre, y en los curiosos y aficionados experimentales de toda la vida, esto es, aquellos que antaño exploraron por ejemplo los temas de radioaficionados o la creación de impresos en formato zine para salirse de las versiones oficiales y ahora instalan sistemas operativos libres o extienden la vida útil de sus aparatos para evitar la obsolescencia programada.

Pero la agencia no es competencia exclusiva de los humanos. También los objetos tienen agencia (Latour, 2008). No porque tengan voluntad sino porque nos interpelan. Las tecnologías, desde el lápiz hasta las gafas de realidad virtual, proceden

3 En inglés, DIY $=$ Do it yourself. DIW0 $=D_{0}$ it with others. DIT0 $=$ Do it together 
como actantes ya que nos configuran, condicionan y determinan tanto a nivel cognitivo y social. Ponen su sello en nuestra subjetividad. Así, las tecnologías no son ajenas a nuestra naturaleza, sino que antes son inseparables de ésta. En contravía del prisma cartesiano, que fomenta los dualismos, las escisiones abismales y los estudios con miradas aisladas, es menester considerar tanto a los sujetos, con su dimensión subjetiva y experiencial, como las tecnologías, que median y cincelan (Rueda Ortiz, 2014). La relación es de doble vía y sin inclinaciones de balanza. Estamos inmersos en contextos históricos, sociales y tecnológicos específicos, situados en complejas redes materiales que son a la vez habitadas y modificadas por nuestras propias experiencias y acciones independientemente de las valoraciones negativas o positivas que se hagan al respecto. Las personas no estamos separadas de las cosas (Esposito, 2016).

Si algo fomenta Platohedro es la participación de los asistentes independientemente del perfil de estos y los resultados que se obtengan. Es decir, no aplican filtros ya que no importa la edad, el género, la escolaridad y los saberes previos que se tengan. Intentan democratizar la batuta de las sesiones al tiempo que buscan difuminar la distancia entre los expertos y los profanos. Hay un potencial político y epistémico latente en esas dinámicas de participación. Político porque crece la toma de poder, en términos de elección y performatividad, de parte del ciudadano común; y epistémico porque se tiene la posibilidad de (re)crear nuestra realidad, interpelarla y configurarla independientemente de las gramáticas impuestas desde otros frentes ${ }^{4}$.

Además, las actividades que se proponen desde Platohedro revitalizan la agencia a través del bricolaje. En efecto, se salen del libreto que usualmente se suele manejar en espacios como la escuela ya que apuestan, entre otras, por revalorizar el trabajo manual y artesanal en oposición al excesivo verbalismo de las aulas, a los cruces de experiencias intergeneracionales, por reconocer saberes (no) expertos, dispersos en un lugar común, profesores

4 Por ejemplo, véase http://platohedro.org/manga-libre/ que devienen estudiantes y viceversa, y valoran el rol epistémico del hacer y el error. También utilizan insumos disponibles en otros ámbitos (repositorios en GitHub, tutoriales alojados en YouTube, páginas de auto creación como Instructables o archivos en Archive.org) y distribuyen sus creaciones para que puedan ser revisadas, criticadas, apropiadas o utilizadas por otros (liberación de contenidos usando licencias abiertas, publicación en tiempo real de los eventos que se realizan o memorias efímeras de las iniciativas que se llevan a cabo).

\section{Socialización}

Aunque el término cultura participativa impera cuando se habla del auge de la tecnología digital, otros autores hablan de cultura de la conectividad ya que sostienen que el reciente ecosistema de plataformas no solo ofrece mayores posibilidades de conexión, interacción y participación en línea, sino que estructuran la propia socialización. Esto sucede al tiempo que cambian su arquitectura de acuerdo con las tendencias de uso de los mismos usuarios. Pasamos de la comunicación en red a la socialización por plataformas (Van Dijck, 2016). Si hay un tema recurrente en las noticias, la vida cotidiana y los estudios académicos es el de cómo internet nos cambió la vida. Tanto se ha dicho sobre sus bondades y perjuicios que parece difícil que surja una perspectiva novedosa. Sin embargo, La cultura de la conectividad hace un aporte original sobre una cuestión que en muchos aspectos sigue siendo muy opaca y requiere, sin dilación, un abordaje sistemático. José van Dijck construye una historia crítica de las redes desde su surgimiento en la primera década del siglo xxi hasta la actualidad. Con notable solvencia, propone un recorrido por las principales plataformas (Facebook, Twitter, Flickr, YouTube y Wikipedia.

Si se usa una categoría clásica de los estudios antropológicos, esta socialización hace parte de una cultura prefigurativa, esta es, una cultura en la que los adultos aprenden de los jóvenes y que se caracteriza por marcar una ruptura generacional con las precedentes, a saber, la postfigurativa y la 
cofigurativa. La postfigurativa es aquella en la que el futuro de los niños está por completo plasmado en el pasado de los abuelos ya que la vida y saberes de éstos son estables, inmutables e imperecederos. En la cofigurativa el modelo de conducta se ofrece a través de los pares y tanto los adultos como los jóvenes aprenden de sus contemporáneos. En cambio, en la prefigurativa no hay guías, las instituciones están en crisis, los pares están también aprendiendo en tiempo real de esta cultura y los saberes del pasado ya no actúan como faros precisos de los caminos venideros (Mead, 1997). Es decir, se están explorando otras maneras de vivir juntos, experimentando con espacios extitucionales y naturalizando formas alternativas de ser y de conocer. En suma, de socializar.

Entre otras, la socialización actual rescata la empatía, lo afectivo, las relaciones, los cuidados y la hospitalidad que se habían menguado en parte por el monopolio del discurso moderno que enarbolaba para el sujeto valores racionales, individuales, autónomos y distantes. Siguiendo a Marina Garcés (2013), hoy se busca recuperar la idea de mundo común que no es el proyecto particular de nadie, sino que es el espacio que comparten personas con historias distintas. Involucrarse en la vida ya que es un problema/oportunidad común. Por ello, como rasgo de este nuevo socius, cada vez sorprende menos el hecho que los cuerpos se distancien de los discursos y realicen lo que las palabras todavía no saben decir.

En suma, la socialización gira sobre el estar juntos. Sobre el cómo se configura la sociedad al tiempo que moldea la subjetividad. Con la diferencia que esa configuración social no se da en el vacío sino en paralelo con el desarrollo de las tecnologías digitales. Hay una influencia recíproca entre los medios conectivos y las personas. Tanto los unos como los otros se configuran entre sí. Se crean dispositivos y a la vez ellos condicionan a las personas. Ese baile en pareja se ubica en una posición equidistante de los clásicos dualismos que imperan en las discusiones sobre sociedad, educación y tecnología. Pero también refuerza el carácter prefigurativo. Por ello al interior de estas prácticas, en este caso Platohedro, se prototipan futuros, se crean formas de proceder que generan ruido en la institucionalidad dominante, se ensayan otras formas de estar, se sospecha de los viejos discursos y las jerarquías sobre el papel, se ponen a circular dominios casi que vetados y se aprende sobre la marcha ${ }^{5}$. También, al proponer talleres sobre, por ejemplo, el navegador TOR y de seguridad digital, buscan subrayar la cara oscura de esa socialización en plataforma, esto es, la vigilancia colateral y el uso mercantil de nuestros intercambios sociales y comunicaciones en el que las plataformas digitales siguen las lógicas capitalistas y de mercado.

\section{Farmacón}

El pensador francés Bernard Stiegler ha sostenido que la técnica es siempre lo impensado. Impensado porque la técnica, que es inherente a los humanos, siempre debe atenderse, cuidarse y reflexionarse ya que a través de ella se (re)crea y reinventa el mundo circundante. Por ello, insiste en la condición farmacológica de la misma, esto es, aquello que "permite cuidar y aquello de lo cual hay que tener cuidado -en el sentido de poner atención a algo: es un poder curativo, en la medida o exceso en que es un poder destructivo" (Stiegler, 2015, p. 30, énfasis del original). Así, toda tecnología es, al tiempo, remedio como veneno.

Stiegler presenta un consejo frente el farmacón, a saber, asumir la adopción antes que la adaptación. La segunda es obligada mientras que la primera es libre. Según su argumento, cuando uno se adapta de manera forzada a una nueva técnica (farmacón), uno se proletariza, se subyuga y expande su toxicidad. La adopción, en cambio, implica elegir y decidir, o en sus términos, es la "condición de individuación del ser farmacológico - de cómo el veneno puede devenir remedio" (Stiegler, 2015, p. 259). Adoptar es emanciparse, adaptarse es someterse.

Es necesario recordar la condición farmacológica de la técnica. Tanto porque es un antídoto contra las miradas reduccionistas que apelan al optimismo

5 Por ejemplo, véase http://platohedro.org/d-formacion/ 
o negativismo acrítico como porque expone las cartas sobre la mesa al subrayar el carácter bifronte de la tecnología digital. En efecto, esta es remedio y veneno. Liberadora y dañina. Las dos cosas al tiempo. Por ejemplo, en varios eventos programados por Platohedro, se hablaba de privacidad, la red profunda, manejo de datos, programas libres y libertad de expresión al tiempo que se usaban diversos programas informáticos para guardar, difundir $y$ enriquecer las sesiones o se desbarataban los dispositivos para conocer su lógica interna. Criticaban la tecnología al tiempo que la usaban ${ }^{6}$. No hablaban de ella desde un escritorio o púlpito sino desde sus mismas entrañas, adoptándola, no adaptándose. De ahí que desarmaran los aparatos, accedieran a los códigos base, modificaran lo que quisieran y aprendieran diseccionando sus insumos de trabajo (véase figura 2). Pura soberanía tecnológica en práctica.

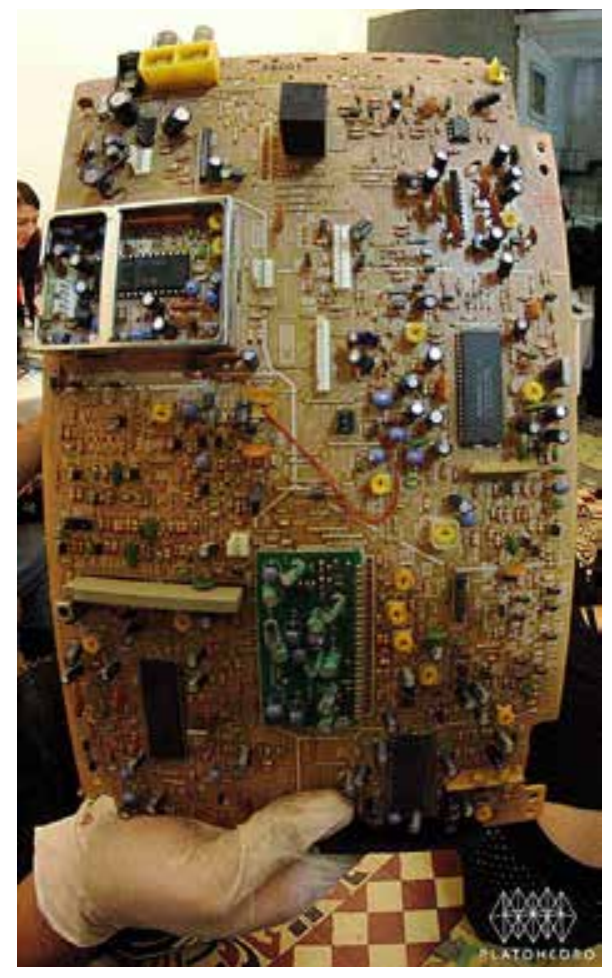

figura 2. Conociendo las entrañas de los computadores. Fuente: Archivo Platohedro

\section{Decolonialidad}

Lo que hace el denominado giro decolonial es pensarse la sociedad latinoamericana desde la diferencia, la diversidad, la praxiología propia, el mestizaje, la hibridez y lo indígena, y no desde la carencia o la falta de desarrollo. En vez de forzar corpus teóricos foráneos para que den cuenta de nuestras dinámicas específicas, valoriza las agendas que tienen en cuenta las particularidades históricas y culturales de la realidad latinoamericana (por ejemplo, el Buen Vivir, el Buen Conocer, el procomún, la investigación acción, entre otros). En palabras de Sierra Caballero (2016):

\section{[...] se trata de tomar en serio el diálogo de saberes y asumir la diferencia de la modernidad evitando cierto dominio eurocéntrico hegemónico -en la actualidad básicamente angloamericano- para afirmar una suerte de ethos barroco como pro- grama científico vital para la región (p. 12, énfasis del original)}

Lo anterior implica reconocer, valorar y aplicar otras formas de vida, otras maneras de producir saberes y de legitimarlos, otros lenguajes y otras formas de representación que son a menudo ignorados por la academia gracias a unos prejuicios epistémicos fuertemente anclados.

Los ricos y variados ejercicios educativos, comunicativos y culturales, que se han gestado en nuestro territorio, invitan a la reflexión y posibilitan la elaboración de constructos teóricos con un fuerte anclaje en lo concreto y en las prácticas que allí se gestan. En lo conceptual, temáticas asociadas a lo popular, lo cotidiano y lo común, de gran riqueza comunicativa, cultural y educativa, podrían explorarse con mayor ahínco. En cuanto a la metodología, estrategias sensibles a estas realidades, tales como la investigación-acción, los enfoques feministas, entre otros, tendrían mayor cabida ya que ofrecen herramientas deconstructoras de las lógicas coloniales para abordar este tipo de fenómenos sociales.

Este ejercicio de decolonialidad posibilitaría miradas alternativas sobre el mundo, particularmente desde el contexto universitario, que van 
más allá del modelo epistémico desplegado por la modernidad occidental. En el modelo se privilegia un punto de vista supuestamente neutral en el que se logra la mayor objetividad posible, el ojo de dios, el meta punto de vista, la hybris del punto cero en palabras de Castro-Gómez (2010). Fuera de esta ficción del distanciamiento, algunas de estas nuevas rutas impulsan lo contrario: la cercanía, el contacto y la implicación en algún nivel. Por ejemplo, la transdisciplinariedad busca cambiar la lógica excluyente (esto o aquello) en favor de una lógica inclusiva (esto y aquello) que promueva la simbiosis entre disciplinas al tiempo que invita no ver la realidad de manera fragmentada y separada por compartimientos, como si el mundo tuviera siempre una lógica cartesiana, dualista, binaria y reduccionista. O la transculturalidad que intenta promover un diálogo de saberes entre las cosmovisiones dominantes y aquellas prácticas y saberes que fueron excluidas del relato moderno de corte occidental por ser, según la oficialidad, míticos, folclóricos, supersticiosos, prerracionales o mera doxa.

Siguiendo de nuevo los planteamientos de Castro-Gómez, es imperativo subrayar que este discurso decolonial no conlleva de entrada, por una parte, ningún tipo de cruzada contra Occidente para reemplazarlo por algún nacionalismo, reducto latinocéntrico o discurso exclusivamente latinoamericano. Por otra, tampoco se busca estar de espaldas a la ciencia moderna o impulsar un nuevo oscurantismo. No se trata de superar las categorías modernas, de ir más allá de las mismas, de rebasarlas o negarlas. Se trata es de ampliar el campo de visibilidad abierto por la ciencia moderna occidental, de incluir dominios tradicionalmente prohibidos como las emociones, la intimidad, el sentido común, lo experiencial, lo ancestral, lo corporal, entre otros. Se pregona es la conjunción epistémica, no su disyunción (Castro-Gómez, 2007, p. 90).

En resumen, la perspectiva decolonial rescata lo local, pone en tela de juicio los meta relatos imperantes y amplía el espectro epistémico para que no se quede atado exclusivamente a lo cognitivo. Platohedro, con sus talleres e invitados, se acerca a narrativas propias y locales, tales como los movimientos indígenas y los saberes populares; están poniendo en duda los lineamientos entusiastas, deterministas, acríticos e imperantes derivados de Silicon Valley en relación con las tecnologías, y favorecen lo corporal, lo afectivo y la filia antes que lo meramente conceptual o curricular. Así el término sea rara vez sea usado por ellos, Platohedro está decolonizando el campo y enriqueciendo la mirada estándar que se tiene frente la cultura digital y la educación. Para la muestra, basta ver talleres que giraban sobre la básica transmutación, el tecnoshamanismo, entre otros, que retomaban temas vinculados tanto a la cultura libre como a los saberes ancestrales y los estudios feministas de la ciencia.

\section{Conclusiones}

Gerd Baumann (2001) habla de la cultura como algo esencialista y procesual. En el primer caso, la cultura actúa como una fotocopiadora que imprime una herencia colectiva que se traduce en un catálogo de ideas, valores y prácticas que configuran la vida y los pensamientos de un pueblo, colectivo o nación. En el segundo caso, la cultura existe en la medida que se actúa, ejecuta y se ejercita, o sea, es performativa e implica un cambio cultural ya que solo tiene sentido en el acto, mientras se lleva a la práctica.

Desde ese prisma, la denominada cultura digital es esencialista y procesual. Si bien se comparten una serie de valores, normas y códigos sociales por parte de un grupo de personas que están inmersos en una sociedad mediada por la tecnología digital, también esa gama de valores, normas y códigos se renegocian y cambian de manera permanente, se entrecruzan con otros paradigmas e identidades culturales y se imposibilita con ello reducir la cultura digital a una categoría esencialista, sin matices y cambios. Al contrario, se revisita de manera permanente.

En el contexto de este escrito, la cultura digital no es algo que solo acontece en un escenario de pantallas o está ajeno de la cultura sin adjetivos. No es un triple ex, es decir, una cultura externa, extraña y exclusiva a una élite. Al contrario, la cultura digital se hibrida con la cultura logrando que ambas se 
retroalimenten de manera permanente. Dicho con más precisión, lo que los humanos llamamos cultura está constituido por elementos dispares, pero constitutivos de nuestra identidad, tales como las técnicas, los artefactos, los lenguajes, las creencias, las normas, entre otros (Monterroza, Escobar y Mejía, 2015). Es la suma de nuestras experiencias humanas. Por ello, la iniciativa que se presentó, Platohedro, no es solo visible en un ámbito virtual o por virtud de un aparato/tecnología/plataforma específica. Tampoco es novedosa en el sentido de surgir exclusivamente por la aparición de lo digital. Es, eso sí, una práctica humana que se manifiesta, se resignifica y dialoga a la luz de las configuraciones mediáticas que se presentan hoy en día. Para la gente de Platohedro, la cultura digital no es un objeto de estudio, sino que es un elemento que se apropia y se integra a su ethos.

Desde lo educativo, las categorías emergentes dejan al menos tres moralejas. La primera es que ponen sobre la mesa la analogía de la relación entre un mapa y el territorio. O sea, el mapa no es el territorio. Esta idea subraya la distancia que hay entre un objeto y su representación. Ambos no son lo mismo. En este tipo de espacios se aprende a crear mapas mientras se recorre el territorio. $\mathrm{Al}$ invitar a descubrir y recorrer tierras vírgenes y en algunos casos inhóspitas para muchas personas, éstas devienen en cartógrafos en tiempo real. Por ejemplo, se aprende sobre programación mientras se programa o se aprende sobre tecnología mientras se desarman equipos tecnológicos propios. Antes que acumular mapas de territorios extraños y ajenos, léase guías, tutoriales o rutas estandarizadas, se busca elaborar cartografías propias a través del cuerpo, el tacto, el error y el diálogo. De esta forma, el aprendizaje se guía no por supuestas respuestas genéricas, recetarios foráneos y lugares cómodos, sino sobre preguntas puntuales que se trabajan sobre el terreno y están vinculadas al contexto.

La segunda moraleja es sobre el aprender con el cuerpo. En Platohedro, y otros espacios similares, se insiste en que el aprendizaje gira sobre la acción. Predomina el cuerpo, no el verbo. Es importante tocar, sentir, manipular, desbaratar, involucrarse con la materialidad y dejarse afectar por las cosas. Dejar que la mano estimule la cabeza y no al contrario ya que también es posible representar y aprender sobre el mundo a través de la carne. Al rechazar tácitamente el prisma dominante del dualismo ontológico, rechazan que lo mental está separado del cuerpo y son escépticos frente al relato educativo que menosprecia la práctica y promueve el cultivo exclusivo de lo mental a través de conocimientos verbales y formales.

La tercera moraleja es sobre el aprender con otros. Dentro el mobiliario de Platohedro imperan las mesas tipo comensal que permiten el diálogo cara a cara y el intercambio de ideas. No hay pupitres ni púlpitos ya que buscan emular más la cocina que el aula de clase. ¿Por qué esta configuración? En parte, porque hay actividades compartidas que se buscan resolver de manera conjunta y porque el diálogo cercano, cara a cara, es central. Y no solo cuando se trata de hacer o deshacer algo. También en los espacios de conversación se estimula esa sensación de cercanía que en algunos casos es acompañada, como en las cocinas familiares o de amigos cercanos, de comida y cerveza. Las prácticas educativas expandidas promueven el aprender con otros porque funcionan con la lógica del proyecto, del intercambio de ideas, el aprendizaje entre pares y la resolución conjunta de problemas. Pero también por razones más mundanas tales como el ahorro de recursos y la mera empatía. En cualquier caso, no funciona la lógica del aprendiz ermitaño o egoísta.

Para cerrar, una reflexión de una de las coordinadoras,

Platohedro es una casa que uno puede ir adaptando, tú llegas acá y sabes que puedes cambiar la mesa de lugar, casi que lo intuyes porque el lugar está todo apropiado, está ensuciado por la gente, en el buen sentido. En otros sitios eso no se puede hacer y no es que esté mal, es otro tipo de características, son espacios en el que si algo se rompe lo vas a tener que pagar y porque responden a otra lógica de intercambio (Luciana, comunicación personal, 23 de junio de 2016). 


\section{Referencias}

Baumann, G. (2001). El enigma multicultural. Un replanteamiento de las identidades nacionales, étnicas y religiosas. Barcelona: Paidós.

Castro-Gómez, S. (2007). Decolonizar la universidad. La hybris del punto cero y el diálogo de saberes. En S. Castro-Gómez, y R. Grosfoguel (Eds.), El giro decolonial: reflexiones para una diversidad epistémica más allá del capitalismo global (pp. 79-91). Bogotá, D.C: Siglo del Hombre Editores: Universidad Central, Instituto de Estudios Sociales Contemporáneos, IESCO-UC: Pontificia Universidad Javeriana, Instituto de Estudios Sociales y Culturales, Pensar.

Castro-Gómez, S. (2010). La hybris del punto cero: ciencia, raza e ilustración en la Nueva Granada (1750-1816). Bogotá: Editorial Pontificia Universidad Javeriana: Instituto de Estudios Sociales y Culturales Pensar.

Charmaz, K. (2008). Constructionism and the Grounded Theory Method. En J. A. Holstein y J. F. Gubrium (eds.), Handbook of constructionist research (pp. 397-413). New York: Guilford Press

Cramer, F. (2015). What Is 'Post-digital'? En D. M. Berry y M. Dieter (Eds.), Postdigital Aesthetics (pp. 12-26). London: Palgrave Macmillan UK.

Deuze, M. (2006). Participation, Remediation, Bricolage: Considering Principal Components of a Digital Culture. The Information Society, 22(2), 63-75. https:// doi.org/10.1080/01972240600567170

Díaz, R. y Freire, J. (Eds.). Educación Expandida. Sevilla: ZEMOS98.

Garcés, M. (2013). Un mundo común. Barcelona: Edicions Bellaterra.

Esposito, R. (2016). Las personas y las cosas. Traducido por Federico Villegas. Buenos Aires: Katz.

Fonseca Díaz, A. D. (2011). Educación expandida y cultura digital. Una exploración de proyectos tecnosociales en Colombia. Hallazgos, 8(15), 71-90. http://dx.doi.org/10.15332/s1794-3841.2011.0015.04

García Canclini, N. (2001). Culturas híbridas: estrategias para entrar y salir de la modernidad. Buenos Aires: Paidós

Jenkins, H., Itō, M., y Boyd, D. (2016). Participatory culture in a networked era: a conversation on youth, learning, commerce, and politics (Versión de Kindle). Cambridge, UK; Malden, MA: Polity Press.

Jenkins, H., Purushotma, R., Weigel, M., Clinton, K., y Robison, A. J. (2009). Confronting the challenges of participatory culture: media education for the 21st century. Cambridge, MA: The MIT Press.
Latour, B. (2008). Reensamblar lo social: una introducción a la teoría del actor-red. Traducido por Gabriel Zadunaisky. Buenos Aires: Manantial.

Lessig, L. (2012). Remix: cultura de la remezcla y derechos de autor en el entorno digital. Barcelona: Icaria.

Lévi-Strauss, C (1997). El pensamiento salvaje. Bogotá: Fondo de Cultura Económica.

Mead, M. (1997). Cultura y compromiso: el mensaje de la nueva generación. Traducido por Eduardo Goligorsky. Barcelona: Gedisa.

Monterroza Ríos, Á., Escobar, J. M., y Mejía Escobar, J. A. (2015). Por una revaloración de la filosofía de la técnica. Revista CTS, 10(30), 265-275.

Navas, E. (2012). Remix theory: the aesthetics of sampling. New York: Springer.

Ocejo, R. E. (2017). Masters of craft: old jobs in the new urban economy. Princeton: Princeton University Press.

Ricaurte Quijano, P. (2018). Laboratorios ciudadanos y humanidades digitales. Digital Humanities Quarterly, 12(1).

Rueda Ortiz, R. (2014). (Trans)formación sociotécnica, subjetividad y política. Pedagogía y saberes, 40, p. 11-22. http://dx.doi.org/10.17227/01212494.40pys11.22

Serres, M. (2013). Pulgarcita. El mundo cambió tanto que los jóvenes deben reinventar todo: una manera de vivir juntos, instituciones, una manera de ser y de conocer... Buenos Aires: Fondo de Cultura Económica

Sierra Caballero, F. (2016). Editorial: Comunicación y Buen Vivir. Nuevas matrices teóricas del pensamiento latinoamericano. Chasqui. Revista Latinoamericana de Comunicación, 131, 9-18. https://doi.org/http:// dx.doi.org/10.16921/chasqui.v0i131.2899.g2734

Stiegler, B. (2015). Lo que hace que la vida merezca ser vivida: de la farmacología. Traducido por Nadia Cortés. Madrid: Avarigani.

Van Dijck, J. (2016). La cultura de la conectividad: una historia crítica de las redes sociales. Buenos Aires: Siglo Veintiuno Argentina.

Uribe Zapata, A. (2017). Una revisión de prácticas educativas expandidas de la ciudad de Medellín. Rev. Guillermo de Ockham, 15(1), 139-148. http://dx.doi. org/10.21500/22563202.2757

Uribe Zapata, A. (2018). Concepto y prácticas de educación expandida: una revisión de la literatura académica. El Ágora USB, 18(1), 277-292. http:// dx.doi.org/10.21500/16578031.3456 